Pedreros Bernal, Y. A., y Medina-Arboleda, I. F. (septiembre-diciembre, 2021). Relación entre el estilo cognitivo y las estrategias de elección en jóvenes. Revista Virtual Universidad Católica del Norte, (64), 41-64. https://www.doi.org/10.35575/rvucn.n64a3

\title{
Relación entre el estilo cognitivo y las estrategias de elección en jóvenes
}

\section{Relation between cognitive style and choice strategies in youth}

\section{Yenni Andrea Pedreros Bernal}

Magister en Estudios Estratégicos

Escuela de Ciencias Sociales, Artes y Humanidades, Universidad Nacional Abierta y a Distancia -UNAD-

Bogotá, Colombia

yenni.pedreros@unad.edu.co

Orcid: https://orcid.org/0000-0003-2353-3128

CvLac: https://scienti.minciencias.gov.co/cvlac/visualizador/generarCurriculoCv.do?cod_rh=0001398170

\section{Iván Felipe Medina-Arboleda}

Doctor en Educación

Escuela de Ciencias Económicas y Administrativas, Corporación Universitaria de Asturias

Bogotá, Colombia

ivan.medina@asturias.edu.co

Orcid: https://orcid.org/0000-0003-3209-9204

CvLac: https://scienti.minciencias.gov.co/cvlac/visualizador/generarCurriculoCv.do?cod_rh=0000609862

Recibido: 23 de junio de 2020

Evaluado: 28 de septiembre de 2020

Aprobado: 25 de marzo de 2021

Tipo de artículo: Investigación Científica y Tecnológica

\section{Resumen}

Los vínculos entre el estilo cognitivo y las estrategias de decisión, en contextos de elección económica, no se han explorado en contextos experimentales. En este estudio se evaluó la relación entre el estilo cognitivo y las estrategias de decisión, basadas en las alternativas o en las dimensiones, en una tarea de simulación de inversión económica con alternativas financieras comerciales. Participaron 58 individuos (universitarios y profesionales) que respondieron la prueba de Desenmascaramiento de Figuras Enmascaradas. Posteriormente, reportaron sus 
preferencias de inversión, y en una tarea de simulación de decisión económica fue evaluado el uso de estrategias basadas en análisis de dimensiones o de alternativas. Los resultados arrojaron una relación modesta y tamaño del efecto pequeño $(r=0.209)$ entre el estilo cognitivo y la estrategia de decisión basada en las alternativas, sin que llegara a ser marginalmente significativa (sig .11). Los hallazgos se discuten en el contexto de la autonomía de los constructos, a saber, las estrategias de elección, en cuanto sensibles a la metacognición, no se asocian con la estilística en cuanto rasgo. Por otra parte, se proponen líneas de trabajo para el desarrollo de tareas subsecuentes que incluyan riesgo de pérdida económica en las alternativas.

Palabras clave: Estilo cognitivo; Estrategias de decisión; Inversión; Psicología económica.

\begin{abstract}
The relation between cognitive style and decision strategies in contexts of economic choice have not been explored in experimental contexts. In this study, the relationship between cognitive style and decision strategies, based on alternatives or dimensions, was evaluated in an economic investment simulation task with commercial financial alternatives. Fifty-eight individuals (university students and professionals) participated who answered the Unmasking of Masked Figures test. Subsequently, they reported their investment preference, and, in an economic decision simulation task, the use of strategies based on analysis of dimensions or alternatives was evaluated. The results show a modest relationship and a small effect size $(r=.209)$ between the cognitive style and the decision strategy based on the alternatives; without it reach a marginal significance (sig. 11). The findings are discussed in the context of the autonomy of the constructs, that is, the choice strategies, as sensitive to metacognition, are not associated with the stylistics as a trait. On the other hand, lines of work are proposed for the development of subsequent tasks that include risk of economic loss in the alternatives.
\end{abstract}

Keywords: Cognitive style; Choice strategy; Investment; Economic psychology. 


\section{Introducción}

En psicología se usa la noción de estilo para referirse a rasgos estables en las interacciones psicológicas (emociones, comportamientos, procesos cognitivos, entre otros), que no tienen grandes cambios a través de las diferentes situaciones, y que, entre otras, permiten diferenciar a las personas entre sí. Según Witkin \& Moore (1974), el estilo es un modo de funcionamiento que, integrado entre dimensiones, conforma la personalidad del individuo. Por su parte, Hederich (2010) considera que el estilo es "un conjunto de regularidades consistentes en la forma de la actividad humana que se lleva a cabo, con independencia del contenido, esto es, de los dominios propios de la actividad" (p. 17). Se han planteado diferentes estilos en el dominio cognitivo, por ejemplo, de aprendizaje, de inteligencia, de enseñanza, de liderazgo, entre otros.

Ramiro et al. (2010) señalan que los estilos cognitivos son rasgos estables en el tiempo y que se emplean habitualmente cuando se toman decisiones y se resuelven problemas; en general, diferencian las actividades cognitivas. Hay varias formas de caracterizar los estilos cognitivos, una de ellas es a partir de dimensiones (Hederich y Camargo, 2000a, 2000c); verbigracia, a partir de extremos o polos opuestos: independencia a dependencia de campo, reflexividad a impulsividad, preferencia de modalidad visual versus verbal (Curione et al., 2010).

La formulación del estilo cognitivo dependencia -independencia de campo visual (DIC) se da a partir de los trabajos de Asch y Witkin (como se citó en Vasco, 2000), en la década de los cuarenta, respecto a las diferencias individuales de la percepción de la verticalidad en pilotos de avión. Actualmente, la evaluación del estilo cognitivo (DIC) se hace con la Prueba de Figuras Enmascaradas (en adelante EFT), la cual, según Hederich (2004), mide la velocidad de reestructuración perceptual, principal indicador de la predominancia de la independencia de campo visual en los individuos. La incorporación del EFT, como medida de la dependenciaindependencia visual de campo, llevó a que la dimensión fuese reinterpretada como una habilidad de tipo perceptual para identificar formas que estaban incorporadas dentro de un contexto enmascarador.

La diferenciación psicológica sobre la base de la polaridad independencia y dependencia del campo se extendió hasta involucrar un amplio rango de funciones: aspectos cognitivos, afectivos-emocionales, de contacto social y neuropsicológicos. En este contexto, los estilos cognitivos (como diferenciador psicológico de los individuos), en interacción con otras 
características situacionales e individuales, explicarían parte de las decisiones de las personas, incluidas las económicas. En Colombia, el modelo de estilos cognitivos de independencia y dependencia del campo visual ha recopilado importante evidencia en rendimiento académico, durante la educación básica primaria (Hederich, 2007; Hederich y Camargo, 2000b; HederichMartínez \& Camargo-Uribe, 2015) y universitaria (A. Hernández, 2019); interacción de los estudiantes con la educación soportada por tecnología (López Vargas et al., 2014; SolórzanoRestrepo y López-Vargas, 2019); y recuerdo y emoción, asociadas con publicidad (Vásquez et al., 2018). Sin embargo, aunque inferibles del modelo de base, los vínculos entre los estilos cognitivos y otros constructos y desempeños, en contextos de decisión económica, han sido inexplorados.

En este contexto, el propósito del estudio es evaluar si existe relación entre el estilo cognitivo dependencia/independencia de campo visual y las estrategias de elección usadas en un contexto de simulación de inversión en jóvenes universitarios y profesionales. Se considera que esta exploración aporta información sobre la interacción entre constructos tipo rasgos (como los estilos) y situacionales como las estrategias de decisión; a su vez, aporta información sobre el vínculo de las condiciones ambientales de las tareas de decisión y las condiciones psicológicas de base (duraderas y situacionales) de los consumidores.

\section{Marco teórico}

\section{La diferenciación psicológica a partir del estilo cognitivo}

De acuerdo con la teoría de diferenciación psicológica (TDP) (Hederich y Camargo, 2000c), la diferenciación del individuo, con respecto a los otros y a su medio ambiente, es decir, la existencia de límites entre el "yo" y el "mundo exterior", es una propiedad estructural de todo sistema psicológico. Es posible hallar grados de diferenciación, cuyos extremos corresponden a polaridades de la valoración: (1) la más diferenciada, característica de individuos cuyos límites entre el yo y el mundo exterior están tajantemente establecidos, y (2) la menos diferenciada, para individuos con límites difusos entre su yo y su entorno físico y social (Camargo, 2001).

Esta diferenciación, en lo que respecta la dimensión independencia y dependencia del campo visual, señala que en el extremo dimensional de la independencia del campo visual se denota la tendencia a percibir las partes como elementos discretos del campo; es decir, se percibe 
de forma analítica: se distinguen los constituyentes esenciales y dominantes que conforman una unidad. A su vez, la tendencia a asignar esquemas de información propia y a organizar la información percibida, de acuerdo con un objetivo. En contraste, la dimensión de dependencia de campo visual es la tendencia perceptual a que la organización general del campo (aquello que se percibe) es la dominante, y las partes del campo se experimentan como fundidas en el todo; por tanto, hay una dificultad para separar los elementos constitutivos de información de sus contextos (Curione et al., 2010).

Así, mientras los independientes son individuos que establecen una separación entre sí mismos y el entorno -incluidas las otras personas -, los dependientes se perciben y definen a ellos mismos como parte de un todo que los contiene. Por tanto, los independientes son individuos más autónomos, con preferencia por manejar sus relaciones sociales, con base en objetivos impersonales y no sobre la base de la empatía o la pertenencia a grupos. En cuanto a la otra polaridad, los dependientes son personas fusionadas con su entorno físico o social (límites difusos del yo y el medio), adscriptivos y sociables, con tendencia a manejar sus relaciones interpersonales con base en la empatía, más que sobre la base de objetivos de trabajo (Becerra et al., 2009; Hederich y Camargo, 2000b).

En psicología educativa, en particular en Colombia, el modelo del estilo de independencia o dependencia del campo visual ha mostrado ser pertinente para explicar fenómenos como el rendimiento académico, la interacción con la tecnología y la regulación del aprendizaje. Por ejemplo, en cuanto al desempeño académico, evaluado a través de pruebas estandarizadas, de estudiantes de educación básica en Bogotá, los estudiantes con independencia del campo visual obtienen puntuaciones más altas; sin embargo, los casos en que se presenta menor seguimiento de reglas sociales -más frecuente en estudiantes independientes- se asocia con menores puntajes académicos (Hederich-Camargo \& Camargo-Uribe, 2015). A su vez, López Vargas et al. (2014), también con estudiantes colombianos de educación básica, encuentran que los estudiantes con independencia de campo visual se fijan metas de aprendizaje más exigentes, y se atribuyen a sí mismos la responsabilidad sobre el desempeño académico, más que lo que lo hacen los de estilo cognitivo dependiente del campo visual.

Las posibilidades de afectación del estilo cognitivo se han explorado tanto hacia fenómenos atencionales básicos, de índole perceptual-relacional de eventos, como hacia niveles de formación superiores, como el universitario. En el campo de fenómenos atencionales perceptuales- 
relacionales, Delgado et al. (2014) encontraron asociaciones positivas, estadísticamente significativas para todos los casos, entre los estilos cognitivos y la formación de relaciones de equivalencia (simetría, transitividad y equivalencia). Ahora bien, en el campo de formación universitaria los hallazgos no resultan contundentes, con respecto al papel del estilo y las así llamadas didácticas de movilidad estilística (para una revisión y ejemplar empírico ver A. Hernández, 2019); entre otras, por la interacción de variables diversas, cognitivas y extracognitivas, como las experiencias personales, otras estilísticas y el tipo de carrera (Borzone Valdebenito, 2017), o la modalidad de aprendizaje, por ejemplo, en entornos virtuales (SolórzanoRestrepo y López-Vargas, 2019), los cuales generan un mejoramiento continuo del capital intelectual o aprendizajes significativos de los actores involucrados (Mercado Borja et al., 2019).

Ahora bien, pese a su amplio uso para el estudio de fenómenos académicos y perceptuales, la relación entre los estilos cognitivos y constructos psicológicos situacionales y metacognitivos (controlables por los individuos), como las estrategias de decisión, no ha sido tan ampliamente explorada. Así, por ejemplo, no es claro si las dimensiones estilísticas de independencia y dependencia se corresponden con preferencias por estrategias de decisión específicas, y qué tan flexibles pueden llegar a ser los individuos con respecto al uso de estrategias, aunque no correspondan con las preferidas en términos de estilística

\section{Estrategias de decisión y elección}

De acuerdo con Hederich y Camargo (2000a), a diferencia del estilo cognitivo (DIC), las estrategias son formas de analizar la información que pueden aprenderse y usarse deliberadamente para lidiar con tareas difíciles. Así, mientras que el estilo es estable, los individuos pueden aprender e implementar estrategias fluidas; es decir, que pueden usarse con relativa autonomía del estilo. Sin embargo, así como se pueden aprender, habría un vínculo de coherencia entre los estilos y determinadas estrategias de decisión (Hederich y Camargo, 2000a).

Dentro de los diferentes modelos de clasificación de las estrategias de decisión, está el modelo de Costes-Beneficios. En esta perspectiva, la toma de decisiones lleva consigo un enfrentamiento a mínimo dos opciones de elección. El dilema de la situación se resolvería a partir del uso de diferentes estrategias, que pueden modificarse situacionalmente, de acuerdo con el 
esfuerzo cognitivo (costo) que exige la evaluación de las consecuencias (beneficio) de las alternativas (Iglesias, de la Fuente, García et al., 2001).

De acuerdo con Tapia (1987), algunas estrategias plantean menos presión a la capacidad de procesamiento cognitivo; por tanto, representan un menor costo (esfuerzo) para el individuo. De acuerdo con el balance de costos y beneficios del uso de las estrategias se seguirá la elección de estas en una situación de decisión particular. Dado que en el proceso de toma de decisión está inmersa la incertidumbre, puesto que no existe una probabilidad constante en las condiciones en las que se tomó una decisión, las estrategias se van modificando, en función de las variables involucradas, como el contexto, la tarea y del propio decisor (Iglesias, de la Fuente y Marín, 2000).

Una forma de clasificar las estrategias de decisión es según el tipo de procesamiento que requieren: basada en el análisis de las dimensiones versus análisis basado en las alternativas. Así, en el análisis de dimensiones, primero se evalúan los valores de un mismo atributo en las diferentes alternativas, y después se consideran, del mismo modo, los demás atributos; el análisis finaliza cuando la diferencia acumulada de las alternativas supera algún criterio establecido, sea subjetivo o externo. En el procesamiento centrado en las alternativas, se evalúa cada alternativa globalmente, antes de evaluar la segunda alternativa (y así sucesivamente) para escoger la alternativa más valorada (Iglesias, de la Fuente, García et al., 2001).

El procesamiento por alternativas parece estar en el contexto natural de las personas, en la forma en que venden y ofrecen bienes y servicios; además, promueve que las personas tomen decisiones bajo este análisis; sin embargo, el análisis por dimensiones se puede adaptar mejor a situaciones según el tipo de elección (Russo \& Dosher, 1983). Con propósitos analíticos, las estrategias se consideran de igual ponderación (basado en alternativas), puesto que todas las dimensiones de las alternativas aportan por igual a los beneficios; y de la suma de las diferencias, en la que se contrastan los beneficios de las alternativas en diferentes dimensiones, y se selecciona la que obtiene en el agregado el mayor valor en diferencia (Bettman et al., 1990; Iglesias, de la Fuente y Marín, 2000).

Al utilizar alguna de las dos estrategias es importante evaluar el esfuerzo cognitivo requerido para tomar la decisión, porque de este esfuerzo dependería el uso de una u otra estrategia. Para Bettman et al. (1991), la dificultad en la elección va a depender y aumentar, generalmente, de si el número de alternativas y de dimensiones aumenta, si alguna información del contenido de la prueba es difícil de procesar y si hay incertidumbre entre los atributos y las alternativas. Según 
Russo \& Dosher (1983), existe una relación entre las estrategias empleadas de decisión y el esfuerzo cognitivo; el procesamiento de elección basado en dimensiones o atributos es cognitivamente más fácil que el basado en alternativas en tareas simples. Sin embargo, cuando la tarea de elección se vuelve más compleja, el esfuerzo cognitivo tenderá a aumentar más en procesamiento de dimensiones que en el de alternativas.

\section{Estilo cognitivo y estrategias de decisión y elección}

En términos del vínculo teórico de estilo cognitivo y estrategias de decisión, el análisis centrado en las dimensiones implica el descomponer las alternativas en elementos de contraste; en contraste, el análisis basado en alternativas privilegiaría la valoración de las totalidades. Por tanto, es esperable que haya una preferencia de los individuos independientes hacia el uso de estrategias que valoran las dimensiones y de los dependientes hacia estrategias centradas en las alternativas.

El contexto de validez externo para vincular la relación entre estilo cognitivo y estrategias de decisión es la toma de decisiones económicas. Al respecto, el trabajo de Fail et al. (2019) vincula a los independientes de campo con una mayor capacidad para valorar oportunidades de negocios, en contraste con los dependientes de campo; sin embargo, su trabajo es de carácter teórico. En su estudio, Chang et al. (2016), con 430 inversores profesionales (clientes y agentes) en el mercado accionario de Taiwán, encontraron que los independientes de campo son más aversos al riesgo que sus contrapartes dependientes, en decisiones que se toman con información no numérica $(p<.001)$; y que prefieren el formato de información de decisiones financieras con una estructura de altos contenidos numéricos; pero que sus diferencias en aversión al riesgo no son significativas en situaciones de bajo riesgo.

En situaciones de evaluación de alternativas de consumo con población general, en Colombia, Vásquez et al. (2018) encontraron indicios sobre comportamientos diferenciales basados en la estilística, en términos de sensibilidad a las rutas de persuasión, el recuerdo del contenido publicitario y el uso de dispositivos tecnológicos. Los participantes con mayor independencia del campo visual recordaron más elementos de los avisos, que aquellos que fueron expuestos y tuvieron mejores desempeños en pruebas de usabilidad de los dispositivos. El estudio no aborda la elección entre alternativas, sino la tendencia a interactuar de manera diferente, tanto con las piezas publicitarias como con el producto; es decir, el aporte de las características 
psicológicas (los estilos) a cómo se da la experiencia con el producto (Sandoval-Escobar et al., 2018).

En este contexto, el propósito del estudio es evaluar si existe relación entre el estilo cognitivo dependencia/independencia de campo visual y las estrategias de elección con las decisiones en inversión en jóvenes universitarios y profesionales de Bogotá (Colombia). Se usó una situación de decisión económica contextualizada, en términos de alternativas de inversión, entendiendo esta como los activos o los derechos de propiedad cuyo propósito es mantener el capital o generar una ganancia (Navarrete, 2013); usando alternativas de inversión ampliamente conocidas, como los depósitos a término (CDT), fiducias a la vista, ahorros informales -cadenas-, acciones, inmuebles, muebles para intercambio, fondos agropecuarios/ganaderos, entre otras.

\section{Método}

\section{Tipo de estudio}

La investigación corresponde a un estudio de corte correlacional asociativo, con pretensiones explicativas y correlación entre la relación de las variables (Avendaño et al., 2018; R. Hernández et al., 2014).

\section{Participantes}

La muestra estuvo conformada por 58 personas, 14 hombres y 44 mujeres (75.9\%); la edad osciló entre 18 y 30 años. El criterio de inclusión del estudio fue que los participantes tuvieran un nivel de formación de universitario o superior, con el propósito de aumentar las probabilidades de que conocieran los instrumentos financieros que se incluían en la simulación. En ese contexto, el grado de escolaridad fue de 43 jóvenes universitarios $(74.1 \%)$ y 15 profesionales de la ciudad de Bogotá (Colombia). Los participantes fueron contactados en los centros educativos o por bola de nieve. En todos los casos, se trató de un muestreo por conveniencia.

\section{Instrumentos}


Los datos se obtuvieron mediante la aplicación de tres instrumentos: Prueba de Figuras Enmascaradas (EFT), lista de chequeo Comportamiento de Inversión y la prueba de Estrategias de Elección.

Prueba de Figuras Enmascaradas (EFT). Es un instrumento usado para la identificación del estilo cognitivo en su dimensión de independencia - dependencia de campo. La prueba mide la velocidad de reestructuración perceptual, principal indicador de la tendencia hacia la independencia de campo. En este trabajo se usó la versión adaptada para población colombiana de Hederich (2004), que consta de cinco subpruebas, cada una de las cuales se presenta en una hoja separada. Las subpruebas contienen una figura simple y un conjunto de 10 figuras complejas diferentes. Cada subprueba tiene un tiempo limitado para su resolución (Hederich, 2004). El puntaje varía de 0 a 50. En el trabajo de Hederich (2007) se reporta una confiabilidad para muestras colombianas de .939 (hombres) y .936 (mujeres), con un análisis de subexploración por sumatoria directa en cada ítem de .873 .

Preferencia de inversión. Reporte de preferencia entre alternativas de inversión para identificar el conocimiento y uso de las alternativas en el contexto natural. Consta de ocho alternativas de inversión (CDT -Certificado de Depósito a Término-, fiducuentas, cadenas, acciones, inmuebles/ muebles para venta o arriendo, fondos agropecuarios/ganaderos, entre otros). El participante marca con una " $X$ " las alternativas de inversión que considera de su preferencia. El formulario se propuso para esta investigación, en tanto, aunque no evalúa un constructo psicológico, valida si los participantes conocen las alternativas financieras de inversión o no.

Prueba de estrategias de decisión. La prueba de estrategia de decisión consiste en una tarea de simulación de inversión con ocho alternativas de inversión (los mismos del instrumento de preferencias), presentadas en duplas (cuatro ensayos, una dupla de alternativas por ensayo). La tarea está desarrollada de acuerdo con los postulados de evaluación de las estrategias de decisión, propuestas por Iglesias, de la Fuente, García et al. (2001).

En la simulación se presentan dos alternativas de elección, comparables en 5 dimensiones (Riesgo, Ganancia, Tiempo de retorno, Confianza y Costos), con atributos diferenciadores. La visión esquemática que resume el entorno gráfico de la simulación se presenta en la figura 1. La situación de elección está encabezada por la instrucción "Si tuviera la oportunidad de invertir su dinero indique, entre las dos alternativas presentadas, cuál sería de su preferencia para realizar esta acción. Recuerde que sus respuestas son confidenciales, serán usadas solo con fines académicos". 
Relación entre el

estilo cognitivo
Relación entre el estilo cognitivo | Revista Virtual Universidad Católica del Norte, 64, 41-64 ISSN: 0124-5821 (En línea)

El participante puede ampliar la información de contraste entre las alternativas al dar clic en las intersecciones, en el título "Ver más".

El participante tiene que escoger entre pares de alternativas. En la figura 1 se ilustra el ensayo de "Certificados de depósito a término" o "Fiducuentas". Ahora bien, el propósito no es identificar la preferencia, sino el tipo de estrategia de decisión que se usa en los ensayos. Con ese propósito analítico, el entorno de simulación es sensible a la ruta de navegación de los “clics” que hacen los participantes: vertical (lectura de todas las características de una alternativa, y luego todas las de la otra alternativa) u horizontal (comparación entre alternativas por cada dimensión); y con base en eso identifica la estrategia de decisión. La prueba presenta puntajes de -1 a 1; si el índice tiende $\mathrm{a}+1$ indica que las personas eligen basados en alternativas, y si tiende a - 1 indica que las personas eligen basadas en las dimensiones; valores obtenidos mediante la fórmula de Iglesias, de la Fuente, García et al. (2001) de forma automática:

$\mathrm{N}^{\mathrm{o}}$ de transiciones intraalternativa $-\mathrm{N}^{\mathrm{o}}$ de transiciones intradimensión

$\mathrm{N}^{\mathrm{o}}$ de transiciones intraalternativa $+\mathrm{N}^{\mathrm{o}}$ de transiciones intradimensión

\section{Figura 1}

Ejemplo esquemático de uno de los ensayos de inversión

\section{Certificados de Depósito a Término (CDT)}

\begin{tabular}{l|l|}
\hline Riesgo & Ver más \\
\hline Ganancia & Ver más \\
\hline $\begin{array}{l}\text { Tiempo de } \\
\text { retorno } \\
\text { Confianza }\end{array}$ & Ver más \\
\hline Costos & Ver más \\
\hline & Ver más \\
\hline
\end{tabular}

Nota: Elaboración propia.

\section{Fiducuentas}

Ver más

Ver más

Ver más

Ver más

Ver más

Fiducuentas 


\section{Procedimiento}

Con las personas que aceptaron participar en el estudio, se hizo una aplicación grupal de la Prueba de Figuras Enmascaradas (EFT), que tiene una duración promedio de 10 minutos. Luego, en una segunda sesión de aplicación, cumplimentaron la prueba de estrategias de elección con una duración de aproximadamente 10 minutos, y la aplicación del instrumento de preferencias inversión, con una duración de 2 minutos aproximadamente. En el inicio de cada prueba el profesional explicó cómo diligenciar las pruebas, con acompañamiento durante la sesión, en caso de que surgieran dudas al respecto.

\section{Consideraciones éticas}

Los participantes diligenciaron el consentimiento informado antes del inicio de la investigación. El documento se elaboró de acuerdo con las especificaciones de la Ley 1090 del 6 de septiembre de (2006) (Colegio Colombiano de Psicólogos, 2015) que establece la reglamentación y los principios éticos para el ejercicio de la psicología. El consentimiento contempla confidencialidad de los datos personales, la categorización del estudio como de mínimo o nulo riesgo, la posibilidad de los participantes de conocer los resultados obtenidos en las pruebas y la identidad, y condiciones de contacto del equipo de investigación.

\section{Resultados}

El análisis de los resultados se implementó con el programa IBM SPSS Statistics ${ }^{\text {, }}$, versión 23. En primer lugar, la totalidad de los participantes (100\%) respondió que conocían las alternativas de inversión que se presentaron en el instrumento de preferencias de inversión. A su vez, la indagación por una preferencia marcada por alguna de las alternativas, antes de la tarea de simulación, mostró que, si bien todas las opciones eran conocidas, en ningún caso hubo una opción escogida como preferente por más de la mitad de la muestra (ver la tabla 1). En la tabla 1 se presenta la información de las opciones conocidas y preferidas con antelación a la simulación. En este contexto, la totalidad de los registros recuperados fueron incluidos en la siguiente fase. 
Relación entre el

estilo cognitivo
Relación entre el estilo cognitivo | Revista Virtual

Universidad Católica del Norte, 64, 41-64

ISSN: 0124-5821 (En línea)

\section{Tabla 1}

Alternativas de inversión conocidas y preferidas por los participantes

\begin{tabular}{lcc}
\hline \multicolumn{1}{c}{ Alternativa de inversión } & Número de participantes & \% de la muestra \\
CDT & 12 & $21 \%$ \\
Fiducuentas o fiducias & 14 & $24 \%$ \\
Cadenas & 17 & $29 \%$ \\
Compra y venta de acciones & 24 & $41 \%$ \\
Compra y venta de Bienes Inmuebles & 16 & $28 \%$ \\
Arriendo de Bienes Inmuebles & 26 & $45 \%$ \\
Fondos Agropecuarios & 5 & $9 \%$ \\
\hline
\end{tabular}

Nota: Elaboración propia.

En cuanto a los puntajes en las pruebas de figuras enmascaradas, el puntaje mínimo fue de 7 puntos, la puntuación máxima de 49. La puntuación media fue de 28.91, con una desviación estándar de 9.4. En la figura 2 se presenta el histograma de frecuencias de los puntajes de la EFT. En general, los datos se agrupan de forma normal; a saber, en los puntajes extremos la minoría de las frecuencias, y en los puntajes medios la mayoría. El estilo cognitivo de los participantes se determinó por sus puntuaciones en la prueba de figuras enmascaradas. Así, de 0 a 15 corresponden al estilo cognitivo dependiente o sensible al campo visual; de 16 a 34, al estilo cognitivo mixto; y de 35 a 50 al estilo cognitivo independiente del campo visual (Delgado et al, 2014; Hederich, 2007). En el primer grupo se clasifican 4 participantes (60 \%), correspondientes a la clasificación de estilo cognitivo sensible o dependiente del campo visual; en el segundo: 35 (57 \%), correspondientes a la clasificación mixta; y en el tercero: 19 (30\%), correspondientes a la clasificación independiente del campo visual.

Con el propósito de verificar la comparabilidad de la muestra con estudios disponibles en el país de la misma población, se evaluó la diferencia de medias con el trabajo de Delgado et al. (2014). La prueba de diferencias de medias es $t=-1.67, p=.19$, razón por la que se rechaza la diferencia, con respecto a los valores reportados de la variable previamente. 
Relación entre el

estilo cognitivo
Relación entre el estilo cognitivo | Revista Virtual

Universidad Católica del Norte, 64, 41-64

ISSN: 0124-5821 (En línea)

Figura 2

Histograma de frecuencias de los puntajes en la prueba EFT

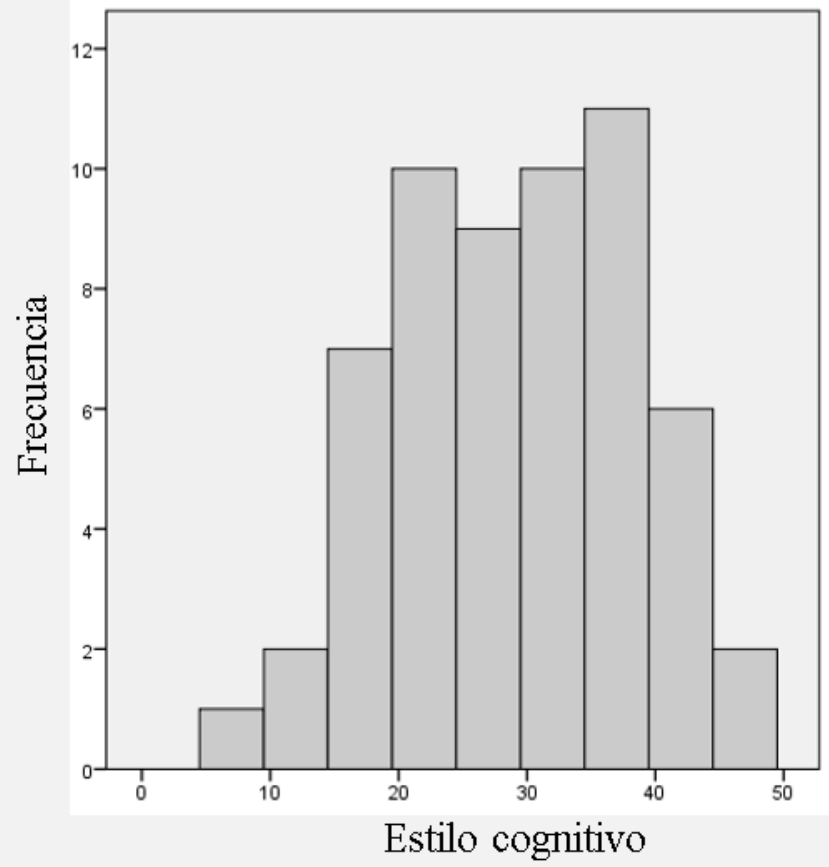

Nota: Elaboración propia.

Para el caso de la prueba de estrategias de decisión, la media fue de .34, con una desviación estándar de .56. En la figura 3 se presenta el histograma de frecuencias de la variable. La distribución de la variable presenta una mayor frecuencia en los valores de 0 a 1 ; es decir, que hay una preferencia en la muestra hacia una de las polaridades de los valores de estrategias de decisión. El análisis de los puntajes extremos -1 a -.5 y de .5 a 1 es ilustrador de tal preferencia: 5 en comparación con 27. Si bien el propósito de la simulación no era identificar cuál alternativa era la preferida, sino registrar el patrón de consulta de información para colegir la estrategia usada, se verificó la cantidad de veces que en la totalidad de ensayos de simulación de elección se escogió la alternativa de la izquierda $(114,49 \%)$ y de la derecha $(118,51 \%)$, de tal manera que no hubo un sesgo de elección relacionada con la posición de las alternativas. En la tabla 2 se presenta, en cada uno de los cuatro ensayos, cuál fue la distribución entre las alternativas. 
Relación entre el

estilo cognitivo
Relación entre el estilo cognitivo | Revista Virtual

Universidad Católica del Norte, 64, 41-64

ISSN: 0124-5821 (En línea)

Tabla 2

Preferencia de elección en los cuatro ensayos (ocho alternativas, dos por ensayo)

\begin{tabular}{|c|c|c|}
\hline Ensayo & Alternativa izquierda & Alternativa derecha \\
\hline 1. CDT versus Fiducias & $31(53 \%)$ & 27 \\
\hline 2. Cadenas versus Acciones & $43(74 \%)$ & 15 \\
\hline $\begin{array}{l}\text { 3. Compra venta bienes inmuebles versus Arriendo de } \\
\text { bienes inmuebles. }\end{array}$ & $24(41 \%)$ & 34 \\
\hline $\begin{array}{l}\text { 4. Fondos agropecuarios versos Compra venta de } \\
\text { bienes inmuebles }\end{array}$ & $16(27 \%)$ & 42 \\
\hline Total & $114(49 \%)$ & $118(51 \%)$ \\
\hline
\end{tabular}

Nota: Elaboración propia.

En este contexto, la exploración gráfica indica que en ambos casos los valores obtenidos en los puntajes superiores son mayores que los obtenidos en los puntajes inferiores. Por tanto, se explora a través de una prueba de correlación el vínculo entre las variables.

\section{Figura 3}

Histograma de frecuencia de la variable estrategia de decisión

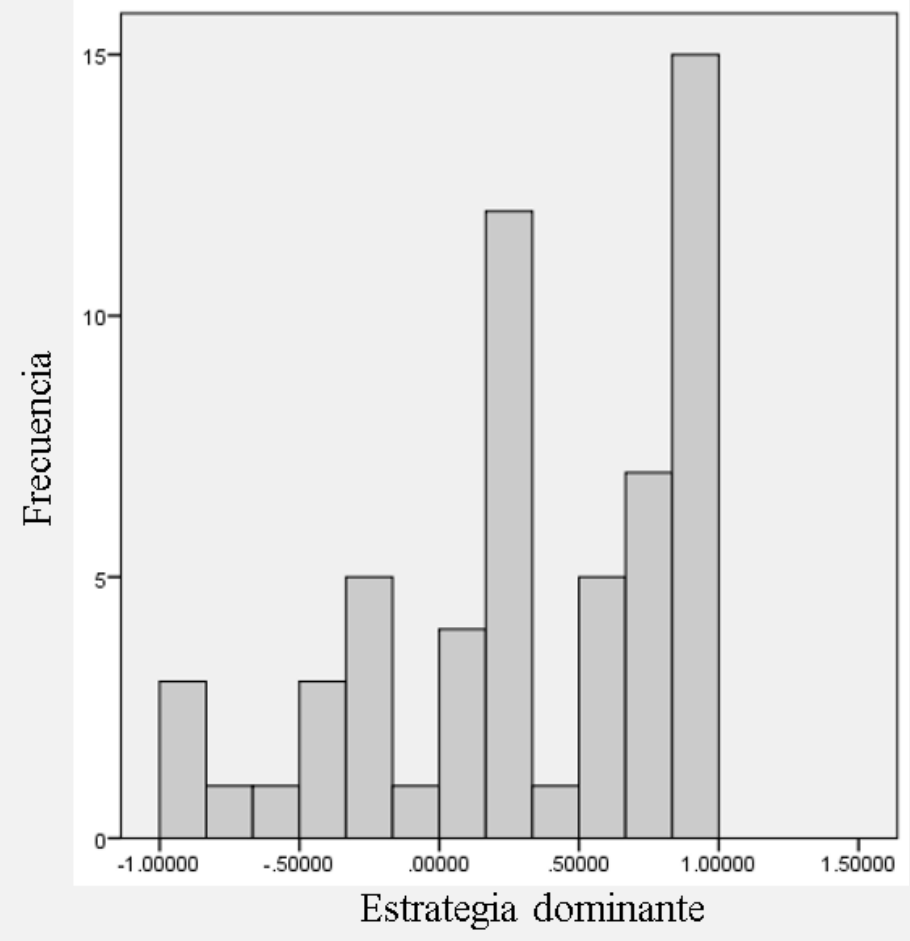

Nota: Elaboración propia 
Relación entre el

estilo cognitivo
Relación entre el estilo cognitivo | Revista Virtual

Universidad Católica del Norte, 64, 41-64

ISSN: 0124-5821 (En línea)

Con el propósito de verificar los supuestos del análisis de correlación con la prueba de $R$ de Pearson se evaluó la normalidad de las distribuciones. Los puntajes de la prueba de figuras enmascaradas y de estrategia de decisión no se diferencian de una distribución normal, dado que los valores de significancia de la prueba Kolmogorov-Smirnov son mayores que $p .05$ (ver tabla 3).

\section{Tabla 3}

Estadísticos de tendencia central y prueba de normalidad para la EFT y la prueba de estrategias de decisión.

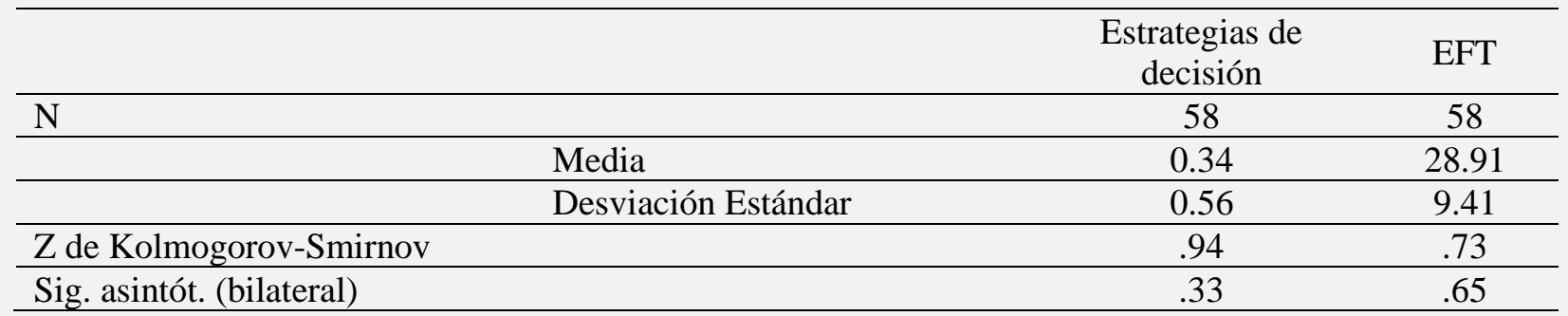

Nota: Elaboración propia.

La correlación de las variables se evaluó a través de la prueba estadística $R$ de Pearson $(r=.209$, sig .11). En términos de la magnitud de la relación de las variables en la muestra, el efecto es pequeño (Field, 2014; Lenhard \& Lenhard, 2016); sin embargo, la probabilidad de cometer un error tipo I, al rechazar la hipótesis nula, es superior $10 \%$; por tanto, aunque cercano a la marginalidad estadística, las consideraciones de generalización de los resultados son limitadas. Así, el vínculo local entre las variables es pequeño, pero no puede extrapolarse a la población.

También, se evaluó la existencia de asociaciones, más allá de lo esperado, entre las preferencias declaradas en los diferentes casos de simulación (cuatro ensayos, ocho opciones) y el estilo cognitivo. Para esto, se usó la transformación de los puntajes de la variable de estilo cognitivo en los tres grupos, dependientes o sensibles de campo en dependiente (tercil inferior), mixtos e independientes (tercil superior). Las pruebas de asociación (valores esperados en distribución equitativa de los casos versus obtenidos) no obtuvieron significancias menores a 0.10. 
En este contexto, se mantiene la hipótesis nula de no asociación entre preferencia en la elección y estilo cognitivo.

\section{Discusión}

El propósito del estudio fue evaluar la relación entre el estilo cognitivo dependencia/independencia de campo (DIC) y las estrategias de elección en una simulación de inversión en jóvenes. Los resultados señalan una relación modesta entre el estilo cognitivo (DIC) y las estrategias de elección (basadas en alternativas o dimensiones); relación que ha de tratarse con cautela para generalizaciones, dado que la significancia, si bien se acerca, no alcanza la marginalidad significativa.

La relación documentada es inversa a lo esperado, por la extensión de la teoría de DIC a la evaluación de alternativas económicamente relevantes. De acuerdo con Fail et al. (2019), la relación entre las medidas obtenidas en los instrumentos habría de ser negativa; es decir, a mayores puntajes en EFT (independencia de campo visual) el puntaje en el tipo de estrategia tendería a - 1, uso de una estrategia centrada en dimensiones.

Los resultados pueden explicarse de dos formas diferentes. La primera, soportada por Hederich y Camargo (2000a), Iglesias, de la Fuente y Marín (2000) e Iglesias, de la Fuente, García et al., (2001), alude a la relativa autonomía de los constructos; a saber, el estilo cognitivo es relativamente constante, pero las estrategias de decisión son situacionales y, por tanto, dependientes de la tarea, más que del individuo. Así, en tareas en las que el número de dimensiones es alto para comparar, la carga cognitiva lleva a cambiar la estrategia a la que requiere menor procesamiento, es decir, basada en la alternativa.

Ahora bien, como lo sugieren los hallazgos de Chang et al. (2016), el aspecto crítico para que se presenten las diferencias en el tipo de estrategia podría interactuar no con la complejidad de la tarea, sino con el riesgo de pérdida de las alternativas que se analizan. En la evaluación de los inversores en una situación simulada, las estrategias usadas por independientes o dependientes de campo interactuaban con el contexto de la decisión; es decir, que en una situación de solo ganancias los estilos cognitivos operan por el uso de estrategias costo eficientes, en términos de análisis (menor esfuerzo cognitivo); pero, en un contexto de posibilidad de pérdidas, los participantes independientes del campo visual usan estrategias analíticas. 
Esta importante diferencia se explicaría, según Kahneman \& Tversky (1979), por la teoría de la Prospección: las personas evalúan las decisiones que tienen que tomar entre esquemas de pérdidas y ganancias, en las cuales muestran aversión al riesgo, cuando se enfrentan a ganancias, y búsqueda de riesgo en el ámbito de pérdida, donde la certeza aumenta la aversión a las pérdidas y la conveniencia a las ganancias. Las predicciones de esta teoría han sido evaluadas con amplia replicabilidad entre muestras (Ruggeri et al., 2020), y se incrementarían de acuerdo con las características psicológicas de los individuos; por ejemplo, el neuroticismo o la extroversión (Oehler, \& Wedlich, 2018) o la independencia y dependencia del campo visual (Chang et al., 2016).

En ese sentido, en cuanto las alternativas de la tarea solo destacaron los beneficios relativos de cada opción, en términos de ganancias, no inducían a la aversión al riesgo, lo cual, al parecer, es más pronunciado en los independientes de campo (Chang et al., 2016). En el trabajo de Coram et al. (2011) se evaluó cómo la presentación de información de tendencia (negativa o positiva) de los estados financieros llevó a estrategias de evaluaciones diferenciales de valoración económica, por parte de avaladores profesionales. Así, los avaladores fueron meticulosos en los casos de estados de pérdida y menos atentos a recabar, analizar y contrastar información contable en los casos de ganancias, pese a su posición de profesionales que, en teoría, tenderían a seguir un estándar de valoración.

En este contexto, una línea de trabajo inmediata con el objetivo de esclarecer el vínculo entre estrategias de decisión, que se ha de explorar en futuras investigaciones, es la de incluir opciones riesgosas, de tal suerte que los escenarios de riesgo queden cubiertos en la tarea experimental.

Por otra parte, aunque la media de la puntuación en EFT se acercó al puntaje central, hay que notar que la mayoría de los participantes en la investigación fueron mujeres. Está bien establecida la importante asociación del género con la estilística cognitiva, por lo que los resultados pueden sesgarse hacia la dimensión dependencia de campo visual (Hederich, 2007). En este ámbito teórico, es importante que futuras investigaciones igualen los géneros participantes, como estrategia indirecta de control de la distribución normal de las dimensiones de estilo en la muestra.

Respecto a las estrategias de elección, el estudio exploró una simulación orientada por la teoría de costo - beneficio (estrategias de igual ponderación y estrategias de suma de diferencias), donde interviene el esfuerzo cognitivo, empleado por Iglesias, de la Fuente, García et al. (2001); 
pero se ignoran en este trabajo variables como el contexto de aplicación y la experiencia con decisiones económicas, dado el programa de formación al que la mayoría de los participantes pertenece. A su vez, no se exploran las limitaciones contextuales ni los factores diacrónicos de la elección, por ejemplo, la experiencia en el manejo de recursos financieros (Gallego et al., 2018) razones que obligan a la precaución en la generalización de los resultados. Por otra parte, este tipo teorías de evaluación de pérdidas y ganancias ha generado una serie de críticas respecto a los supuestos generales de abstracción de las situaciones de elección económica que difieren, en su evaluación empírica, de las circunstancias en las que las personas eligen en su cotidianidad (Vázquez-Parra et al., 2018).

Ahora bien, dada que es una línea de investigación que inicia su evaluación empírica (Fail et al., 2019) no hay puntos de contrastes de tales parámetros metodológicos; pues si bien existen investigaciones sobresalientes frente al estilo cognitivo, en relación con el ámbito educativo, de enseñanza y de aprendizaje, entre muchas otras, no se presentan específicamente sobre la relación con la economía conductual y la psicología del consumidor.

\section{Conclusiones}

El trabajo evaluó de forma pionera para Colombia el vínculo entre el estilo cognitivo de la independencia y dependencia del campo visual y las estrategias de decisión, centradas en las dimensiones o en las alternativas en un contexto de elección económica. El vínculo es modesto y no generalizable; sin embargo, soporta la noción de la interacción entre constructos de rasgo de diferenciación psicológica y estrategias situacionales, y no de la preeminencia exclusiva de alguno de estos constructos como variables psicológicas que afectan la elección económica.

Así, en condiciones en las que el énfasis está en los posibles beneficios -pero, en que en ninguna de las alternativas hay riesgo de pérdida- se privilegia la evaluación centrada en las alternativas (costo eficiencia cognitiva). Esta circunstancia conlleva a que los inversores puedan optar por estrategias de menor carga cognitiva, pues las condiciones de presentación de las alternativas no inducen en la evaluación esmerada (ponderación); por ende, inducirían a elecciones riesgosas en contextos situados. Ahora bien, en futuras investigaciones se evaluará el funcionamiento de tal estrategia cuando los escenarios inducen la aversión a la pérdida y su vínculo con el estilo cognitivo de independencia y dependencia del campo visual. 


\section{Referencias}

Avendaño, B. L., Serrano, S., Toro, R., y Medina-Arboleda, I. F. (2018). Una perspectiva dimensional de las estrategias de análisis utilizadas en la investigación en psicología. En V. Sánchez, M. I. Barreto, y B. Avendaño (Coords.), Investigación en psicología: aplicaciones e intervenciones (pp. 23 - 32). Universidad Católica de Colombia. https://publicaciones.ucatolica.edu.co/pdf/logos-vestigium-investigacion-en-psicologiacato.pdf

Becerra, F., Parra, M., y Vargas, M. (2009). Estilo cognitivo predominante en estudiantes universitarios de nutrición y dietética. Revista de la Facultad de Medicina, 59, 113-124. https://revistas.unal.edu.co/index.php/revfacmed/article/view/23707/24396

Bettman, J. R., Johnson, E. J., \& Payne, J. W. (1990). A componential analysis of cognitive effort in choice. Organizational Behavior and Human Decision Processes, 45, 111- 139. https://doi.org/10.1016/0749-5978(90)90007-V

Bettman, J. R., Johnson, E. J., \& Payne, J. W. (1991). Consumer decision making. In. T. S. Robertson, \& H. H. Kassarjian. Handbook of consumer behavior (pp. 50-84). Prentice Hall. https://www.worldcat.org/title/handbook-of-consumer-behavior/oclc/21910342

Borzone Valdebenito, M. A. (2017). Autoeficacia y vivencias académicas en estudiantes universitarios. Acta Colombiana de Psicología, 20(1), 266-274. http://www.dx.doi.org/10.14718/ACP.2017.20.1.13

Camargo, A. (2001). Lenguaje y estilos cognitivos una aproximación al problema de las diferencias individuales en la actividad lingüística. En J. Bernal (Coord.), Lenguaje y Cognición. Universos Humanos (pp. 49-74). Instituto Caro y Cuervo. https://dialnet.unirioja.es/servlet/libro?codigo $=3374$

Chang, S. C., Tang, Y. C., \& Liu, Y. J. (2016). Beyond objective knowledge: The moderating role of field dependence-independence cognition in financial decision making. Social Behavior and Personality: an international journal, 44(3), 519-527. https://doi.org/10.2224/sbp.2016.44.3.519 
Colegio Colombiano de Psicólogos. (2015). Deontología y Bioética del Ejercicio de la Psicología en Colombia. Manual Moderno. https://www.worldcat.org/title/deontologia-y-bioeticadel-ejercicio-de-la-psicologia-en-colombia-5a-ed/oclc/932334832x

Coram, P. J., Mock, T. J., \& Monroe, G. S. (2011). Financial analysts' evaluation of enhanced disclosure of non-financial performance indicators. The British Accounting Review, 43(2), 87-101. https://doi.org/10.1016/j.bar.2011.02.001

Curione, K., Miguez, M., Crisci, C., y Maiche, A. (2010). Estilos cognitivos, motivación y rendimiento académico en la universidad. Revista Iberoamericana de Educación, 54(3), 19. https://doi.org/10.35362/rie5431671

Delgado, D., Medina, I. F., y Jiménez, L. (2014). Relaciones de equivalencia y estilo cognitivo: hallazgos de una relación no explorada. Suma Psicológica, 21(1), 19-27. https://doi.org/10.1016/S0121-4381(14)70003-7

Fail, C. A., Millam, L. A., \& Reinhardt, M. M. (2019). Field-dependence and independence: does an old theory hold promise for entrepreneurship? Global Journal of Entrepreneurship, 3(1), 11-18. https://go.gale.com/ps/anonymous?id=GALE\%7CA613341441

Field, A. (2014). Discovering statistics using IBM SPSS statistics (4th ed). Sage.

Gallego, D. E., Bustamante, L. E., Quintero, L. F., Jiménez, J. I. y Echeverri Gutiérrez, C. A. (septiembre-diciembre, 2018). La importancia de los recursos financieros personales y su relación con la inteligencia financiera: revisión documental. Revista Virtual Universidad Católica del Norte, (55), 173-191. https://revistavirtual.ucn.edu.co/index.php/RevistaUCN/article/download/1004/1453

Hederich, C. (2004). Estilo cognitivo en la dimensión de Independencia-Dependencia de Campo. Influencias culturales e implicaciones para la educación (Tesis de doctorado). Universidad Autónoma de Barcelona.

Hederich, C. (2007). Estilo cognitivo en la dimensión de dependencia-independencia de campo. Influencias culturales e implicaciones para la educación. Universidad Pedagógica Nacional.

Hederich, C. (2010). Acerca de la noción general de estilo en la educación pertinencia, importancia y especificidad. Actualidades Pedagógicas, $\quad 55, \quad 13-21$. https://ciencia.lasalle.edu.co/ap/vol1/iss55/14/ 
Hederich, C., y Camargo, A. (2000a). Estilo cognitivo en la Educación. Itinerario Educativo. Revista de la Facultad de Educación, 36, 43 -74.

Hederich, C., y Camargo, A. (2000b). Estilo cognitivo y logro académico en la ciudad de Bogotá. Revista Colombiana de Educación, (40-41). https://doi.org/10.17227/01203916.7782

Hederich, C., y Camargo, A. (2000c). Estilos cognitivos en el contexto escolar. Editorial Universidad Pedagógica Nacional

Hederich-Martínez, C., \& Camargo-Uribe, A. (2015). Cognitive style and educational performance. The case of public schools in Bogotá, Colombia. Educational Psychology, 36(4), 719-737. https://doi.org/10.1080/01443410.2015.1091916

Hernández, A. (2019). El papel de la reflexión en el rendimiento académico, la metacognición y el aprendizaje autorregulado en estudiantes con diferentes estilos cognitivos (Tesis de doctorado). Universidad Pedagógica Nacional. http://hdl.handle.net/20.500.12209/10722

Hernández, R., Fernández C., y Baptista, P. (2014). Metodología de la investigación (6 Ed.). Editorial Mc Graw Hill.

Iglesias, S., de la Fuente, E., García, J. G., y de la Fuente, L. (2001). Procesos de decisión con tiempo autoadministrado y limitado: ¿Una respuesta adaptativa a la similitud, al formato y a la experiencia? Anales de Psicología, 17(2), 255-274. https://revistas.um.es/analesps/article/view/28991

Iglesias, S., de la Fuente, E., y Marín, I. (2000). Efecto de las estrategias de decisión sobre el esfuerzo cognitivo. Psicothema, 12(2), 267-272. http://www.psicothema.com/pdf/288.pdf

Kahneman, D., \& Tversky, A. (1979). Prospect theory: An analysis of decision under risk. Econometrica: Journal of the Econometric Society, 263-291. https://www.jstor.org/stable/1914185

Lenhard, W., \& Lenhard, A. (2016). Calculation of Effect Sizes. http://dx.doi.org/10.13140/RG.2.2.17823.92329

López Vargas, O., Ibáñez Ibáñez, J., y Chiguasuque Bello, E. (2014). El estilo cognitivo y la fijación de metas de aprendizaje en ambientes computacionales. Pensamiento Psicológico, 12(1), 133-148. http://dx.doi.org/10.11144/Javerianacali.PPSI12-1.ecfm 
Mercado Borja, W. E., Guarnieri, G., y Rodríguez, G. L. (2019). Análisis y evaluación de procesos de interactividad en entornos virtuales de aprendizaje. Trilogía Ciencia Tecnología Sociedad, 11(20), 63-99. https://doi.org/10.22430/21457778.1213

Navarrete, J. (2013). Principales vectores que determinan la decisión de inversión en las empresas de la región del Maule. Universidad de Talca.

Oehler, A., \& Wedlich, F. (2018). The relationship of extraversion and neuroticism with risk attitude, risk perception, and return expectations. Journal of Neuroscience, Psychology, and Economics, 11(2), 63-92. https://doi.org/10.1037/npe0000088

Ramiro, P., Navarro, J. I., Menacho, I., \& Aguilar, M. (2010). Cognitive style: reflexivityimpulsivity among school children with high intelectual level. Revista Latinoamericana de Psicología, 42, 193-202. http://dx.doi.org/10.14349/rlp.v42i2.471

Ruggeri, K., Alí, S., Berge, M. L., Bertoldo, G., Ludvig, D., Cortijos-Bernabeu, A., Davison, D., Demić, E., Esteban-Serna, C., Friedemann, M., Gibson, S. P., Jarke, H., Karakasheva, R., Khorrami, P. R., Kveder, J., Lind Andersen, T., Lofthus, I. S., McGill, L., Nieto, A. E., ..., $\&$ Folke, T. (2020). Replicating patterns of prospect theory for decision under risk. Nature Human Behaviour, 1-12. https://doi.org/10.1038/s41562-020-0886-X

Russo, J. E., \& Dosher, B. A. (1983). Strategies for multiattribute binary choice. Journal of Experimental Psychology: Learning, Memory, and Cognition,9(4), 676-696. https://doi.org/10.1037/0278-7393.9.4.676

Sandoval-Escobar, M., Medina-Arboleda, I. F., Ávila Campos, J., y Moreno Hernández, L. (2018). Aprendizaje de la marca: aproximaciones conceptuales desde la psicología del consumidor. Suma de Negocios, 9(20), 138-144. https://dx.doi.org/10.14349/sumneg/2018.v9.n20.a8

Solórzano-Restrepo, J., y López-Vargas, O. (2019). Efecto diferencial de un andamiaje metacognitivo en un ambiente e-learning sobre la carga cognitiva, el logro de aprendizaje y la habilidad metacognitiva. Suma Psicológica, 26(1), 37-45. http://dx.doi.org/10.14349/sumapsi.2019.v26.n1.5

Tapia, J. (1987). ¿Enseñar a pensar? Perspectivas para la educación compensatoria. Ministerio de Educación y Ciencia.

Vasco, C. E. (2000). Una teorización cognitiva acerca de la diferencia entre conceptos predicativos o relacionales y conceptos operativos o funcionales. En J. J. Botero, J. Ramos y A. Rosas 
Relación entre el

estilo cognitivo
Relación entre el estilo cognitivo | Revista Virtual

Universidad Católica del Norte, 64, 41-64

ISSN: 0124-5821 (En línea)

(Comps.), Mentes reales: La ciencia cognitiva y la naturalización de la mente (pp. 183202). Siglo del Hombre/Universidad Nacional de Colombia.

Vásquez, L., Sandoval-Escobar, M., Velandia, A., y Hederich, C. (2018) Influencia del estilo cognitivo y la ruta de persuasión sobre las actitudes y reconocimiento de un aviso y la usabilidad de un producto tecnológico. En D. Forero (Ed.), Elección, Razonamiento y Decisión (pp. 76-89). Konrad Lorenz Editores. https://repositorio.konradlorenz.edu.co/bitstream/001/1652/1/3_Eleccion_consumidor.pdf

Vázquez-Parra, J. C., Nieto-Caraveo, E., y García-Caballero, L. (septiembre-diciembre, 2018). Economía y Utilitarismo. La necesidad de una teoría racional apegada a la realidad. Revista Virtual Universidad Católica del Norte, (54), 129-144. https://revistavirtual.ucn.edu.co/index.php/RevistaUCN/article/download/1004/1453

Witkin, H. A., \& Moore, C. A. (1974 April 15 - 19). Cognitive Style and the Teaching Learning Process [Conference paper] 59 $9^{\text {th }}$ Annual Meeting of the American Educational Research Association, Chicago, IL. https://files.eric.ed.gov/fulltext/ED097356.pdf 